of an overarching commitment to limit u carbon emissions. North America's energy challenges would then become a vehicle for beneficial economic coordination and integration rather than remaining a source of rancour and friction.

A key step is a moratorium on new oil-sands development and transportation projects until better policies and processes are in place. Reform is needed now: decisions made in North America will reverberate internationally, as plans for the development of similar unconventional reserves are considered worldwide.

With clearer policy, smarter decisions and stronger leadership, Canada and the United States can avoid the tyranny of incremental decisions - and the lasting economic and environmental damage that poorly conceived choices will cause.

Wendy J. Palen is in the Department of Biological Sciences at Simon Fraser University, Burnaby, British Columbia, Canada. Thomas D. Sisk is in the School of Earth Sciences and Environmental Sustainability at Northern Arizona University, Flagstaff, Arizona, USA. Maureen E. Ryan is in the School of Resource and Environmental Management at Simon Fraser University. Joseph L. Árvai is in the Department of Geography at the University of Calgary, Alberta, Canada. Mark Jaccard is in the School of Resource and Environmental Management at Simon Fraser University. Anne K. Salomon is in the School of Resource and Environmental Management at Simon Fraser University. Thomas Homer-Dixon is in the Balsillie School of International Affairs at the University of Waterloo, Ontario, Canada. Ken P. Lertzman is in the School of Resource and Environmental Management at Simon Fraser University. e-mail:wpalen@sfu.ca

1. Canadian Association of Petroleum Producers. 2014 CAPP Crude Oil Forecast, Markets, \& Transportation: Refinery Data (CAPP, 2014)

2. Rooney, R. C., Bayley, S. E. \& Schindler, D. W. Proc. Natl Acad. Sci. USA 109, 4933-4937 (2012).

3. Kurek, J. et al. Proc. Natl Acad. Sci. USA 110, 1761-1766 (2013).

4. Kelly, E. N. et al. Proc. Natl Acad. Sci. USA 106, 22346-22351 (2009).

5. Chen, Y. Cancer Incidence in Fort Chipewyan, Alberta 1995-2006 (Alberta Cancer Board, 2009).

6. Environment Canada. Canada's Emission Trends (2013).

7. Office of the Auditor General of Canada. 2012 Spring Report of the Commissioner of the Environment and Sustainable Development Ch. 2 (2012).

8. Arvai, J., Gregory, R., Bessette, D. \& CampbellArvai, V. Iss. Sci. Technol. 28, 43-52 (2012).

9. Kenney, L., Bessette, D. \& Arvai, J. J. Environ. Plan. Mgmt http://dx.doi.org/10.1080/0964 0568.2014.899205 (2014).

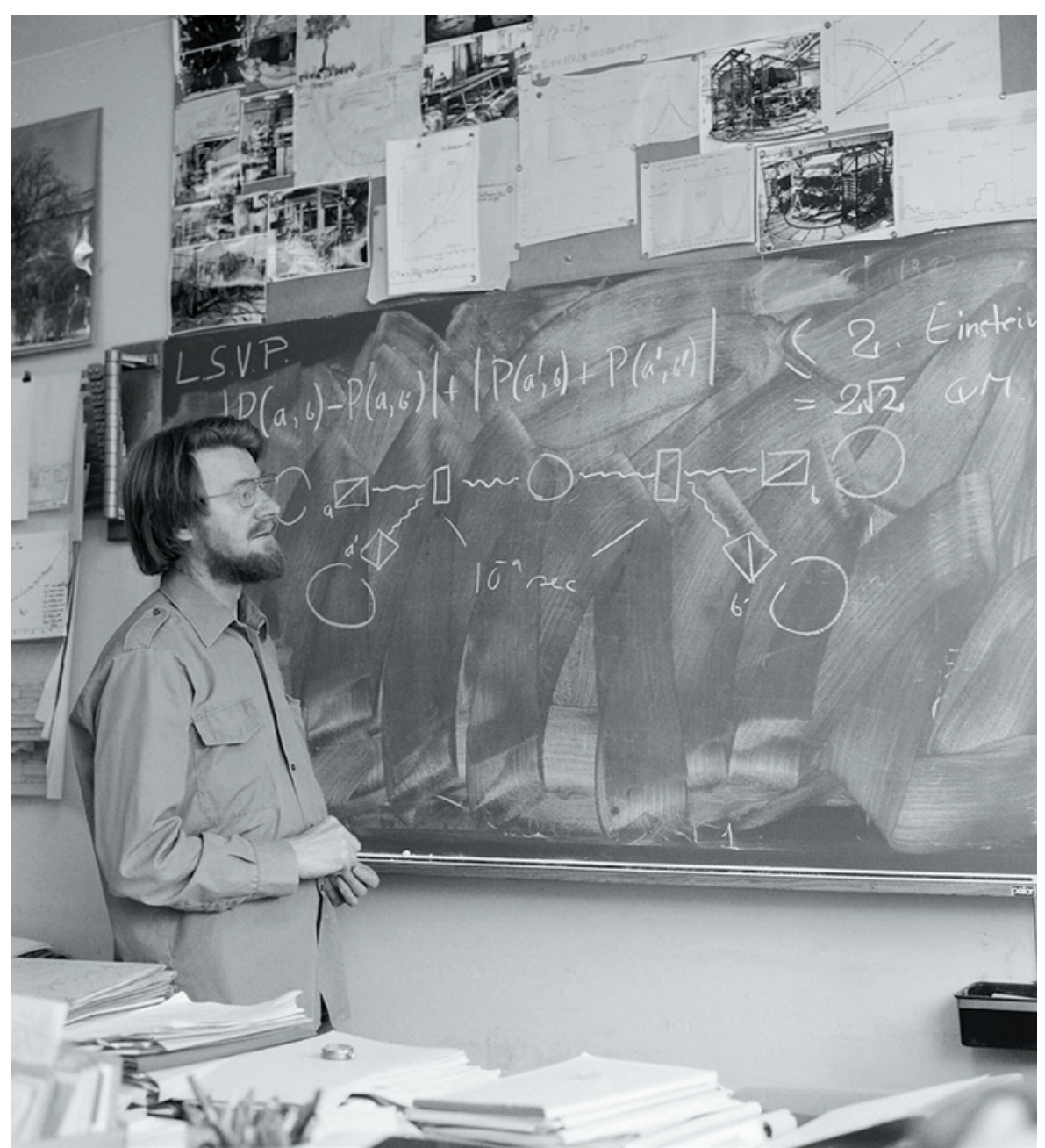

Physicist John Bell at CERN, Europe's particle-physics lab near Geneva, Switzerland, in 1982.

\section{Bell's theorem} still reverberates

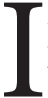
n 1964, Northern Irish physicist John Bell proved mathematically that certain quantum correlations, unlike all other correlations in the Universe, cannot arise from any local cause ${ }^{1}$. This theorem has become central to both metaphysics and quantum information science. But 50 years on, the experimental verifications of these quantum correlations still have 'loopholes', and scientists and philosophers still dispute exactly what the theorem states.

Quantum theory does not predict the outcomes of a single experiment, but rather the statistics of possible outcomes. For experiments on pairs of 'entangled' quantum particles, Bell realized that the predicted correlations between outcomes in two wellseparated laboratories can be profoundly mysterious (see 'How entanglement makes the impossible possible'). Correlations of this sort, called Bell correlations, were verified experimentally more than 30 years ago (see, for example, ref. 2). As Bell proved in 1964, this leaves two options for the nature of 
reality. The first is that reality is irreducibly random, meaning that there are no hidden variables that "determine the results of indi-

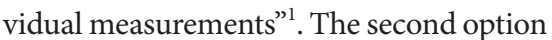
is that reality is 'non-local', meaning that "the setting of one measuring device can influence the reading of another instrument, however remote" .

Most physicists are localists: they recognize the two options but choose the first, because hidden variables are, by definition, empirically inaccessible. Quantum information scientists embrace irreducible randomness as a resource for secure cryptography ${ }^{3}$. Other physicists and

"Before investing too much angst or money, one wants to be sure that Bell correlations really exist."

philosophers (the 'non-localist camp') dispute that there are two options, and insist that Bell's theorem mandates non-locality ${ }^{4}$.

Such views seem contradictory. But I believe that these two camps can be partially reconciled ${ }^{5}$ by delving into what 'causation' means. Doing so reveals the depth of the real principles at stake, the challenges facing each camp, and the future priorities for closing the loopholes in experiments to observe Bell correlations.

\section{FREE CHOICE}

Many localists cite Albert Einstein's 1905 principle of relativistic causality as a reason to reject non-locality. This principle says that causal influences cannot propagate faster than light. That is, one event can cause another (later) event only if they are close enough in space that the 'effect' could have been reached by a beam of light from the 'cause'. But the non-locality option must involve faster-than-light causal influences, contrary to Einstein's principle, for measurements made far enough apart and close to simultaneous. Bell correlations under these circumstances have been observed many times since 1982 (ref. 2), using photons with entangled polarizations.

Although the two camps disagree on whether Bell experiments imply faster-thanlight causal influences, neither think that these experiments allow faster-than-light communication. Faster-than-light communication has never been observed. Its impossibility follows from Einstein's principle of relativistic causality and the following axiom of causation: if an event is seen to depend statistically on a freely chosen action, then that action is a cause of that event.

For example, if a radio comes on when and only when I choose to flip a switch, then my action must cause the sound. Combining this with Einstein's principle (no faster-thanlight causal influences) implies that if I freely choose the time to flip a switch on Earth, a radio on the Moon cannot be expected to come on at that exact time. There must be a delay of at least 1.3 seconds (the time it takes light to travel to the Moon).

It is wrong to argue (as some localists do) that the impossibility of faster-than-light communication rules out non-locality. Locality, as Bell introduced it in 1964, is a stronger concept than no faster-than-light communication. That is, nature could be non-local without allowing faster-thanlight signalling.

For localists to derive locality from the principle of relativistic causality, they need a stronger version of the above axiom of causation: the phrase "is seen to depend on" must be replaced by "depends, in theory, on" 5 . The point is that it may not be possible to see the theoretical dependence if there are other, hidden variables on which the event also depends. This is the case in the versions of quantum theory favoured by the non-localist camp ${ }^{4}$.

\section{ANOTHER THEOREM}

Bell himself was a non-localist, an opinion he first published in 1976 (ref. 6), after introducing a concept, "local causality", that is subtly different from the locality of the 1964 theorem. Deriving this from Einstein's principle requires an even stronger notion of causation: if two events are statistically correlated, then either one causes the other, or they have a common cause, which, when taken into account, eliminates the correlation.

Colloquially, this "principle of common cause" says that correlations have explanations. For example, if you and I never communicate, but one day we both become concerned about a looming war in Ruritania, then there must be a common cause for our thoughts (such as news reports).

In 1976, Bell proved that his new concept of local causality (based implicitly on the principle of common cause), was ruled out by Bell correlations ${ }^{6}$. In this 1976 theorem there was no second option, as there had been in the 1964 theorem, of giving up hidden variables. Nature violates local causality.

It is unfortunate that quantum scientists seldom distinguish the 1976 theorem from the 1964 theorem. It is doubly unfortunate that Bell sometimes used "locality" as shorthand for "local causality", adding to the confusion. Non-localists maintain that the two theorems are the same, that locality is the same as local causality, and thus that hidden variables played no essential part in Bell's 1964 paper $^{4}$. But, as I have shown ${ }^{5}$, these claims do not hold up under careful analysis.

\section{RECONCILING THE CAMPS}

The contradictory claims by the two camps thus arise because they mean different things by 'Bell's theorem' and different things by 'local' (or 'non-local'). For localists, Bell's theorem is the 1964 one, and the preferred choice is to keep locality and forgo hidden variables. For non-localists, Bell's theorem is (or should be) the 1976 one, which leaves no choice but to forgo local causality.

But one can go further, by recalling that local causality rests on two principles: Einstein's principle of relativistic causality, and the principle of common cause. Thus Bell's 1976 theorem can be restated as: either causal influences are not limited to the speed of light, or events can be correlated for no reason.

This, I suggest, is the best way to reconcile the two camps. It enables them to agree on a single Bell's theorem, and what logical options it offers, even if they prefer different options.

Those who insist that correlations are explicable must conclude that causal influences can go faster than light. A challenge for these non-localists is: why does nature nevertheless conspire to prevent fasterthan-light signalling?

Those who hold Einstein's principle to be inviolable (the localists) must conclude that some events are correlated for no reason. A challenge for them is: if correlations do not necessarily imply a cause, when should scientists look for causes, and why?

\section{THE PATH FORWARD}

Bell correlations can be seen as a problem, or an opportunity. They present us with a dilemma; each of the principles at stake (relativistic causality and common cause) underpins a vast mesh of scientific inference and intuition, and yet one must be forgone. But Bell correlations also present us with a marvellous information-technology resource: measurement outcomes that cannot possibly be known to anyone before they occur.

Before investing too much angst or money, one wants to be sure that Bell correlations really exist. As of now, there are no loophole-free Bell experiments. Experiments in 1982 by a team led by French physicist Alain Aspect ${ }^{2}$, using well-separated detectors with settings changed just before the photons were detected, suffered from an 'efficiency loophole' in that most of the photons were not detected. This allows the experimental correlations to be reproduced by (admittedly, very contrived) local hidden variable theories.

In 2013, this loophole was closed in photon-pair experiments using highefficiency detectors ${ }^{7,8}$. But they lacked large separations and fast switching of the settings, opening the 'separation loophole': information about the detector setting for one photon could have propagated, at light speed, to the other detector, and affected its outcome.

There are several groups worldwide racing to do the first Bell experiment with large separation, efficient detection 


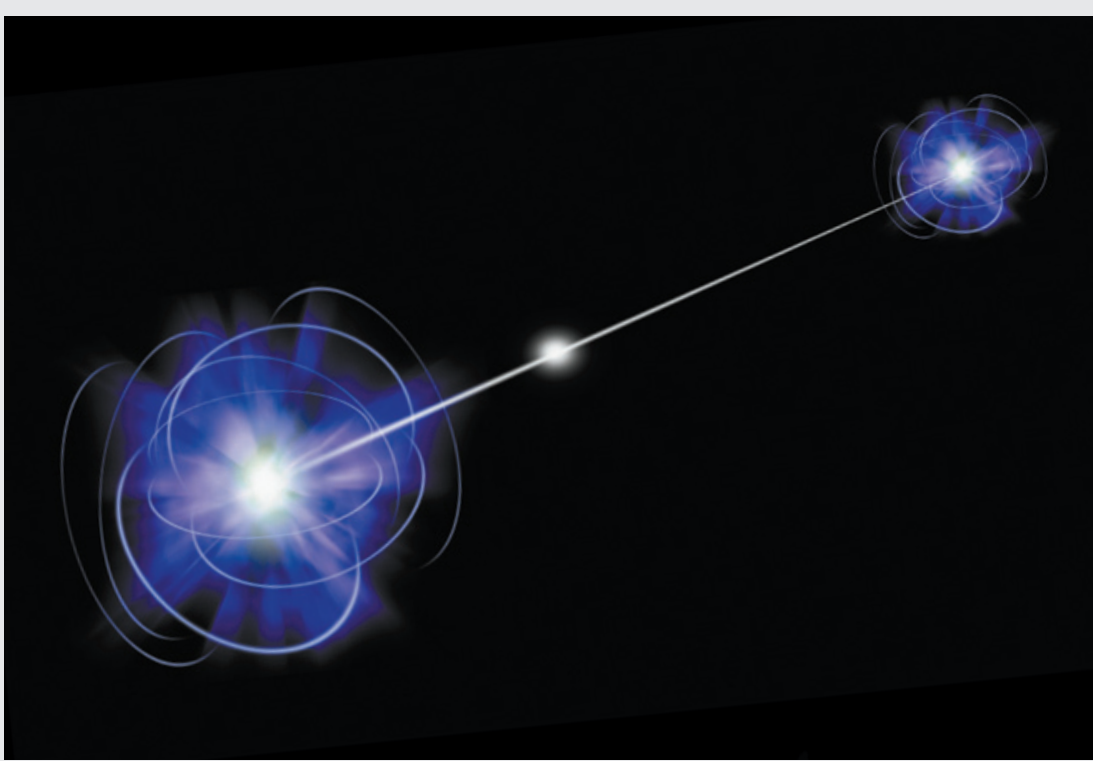

Quantum entanglement can link the quantum states of particles even when they are separated by long distances (artist's impression).

\section{BELL CORRELATIONS}

\section{How entanglement makes the impossible possible}

Consider an impossible square - a square divided into nine smaller squares, each containing a 0 or a 1 , such that the number of $1 \mathrm{~s}$ in every column is even, and the number of $1 \mathrm{~s}$ in every row is odd. Why is this impossible? Because the total number of $1 \mathrm{~s}$, from the columnrule, is even + even + even = even, but the total number of $1 \mathrm{~s}$ from the row-rule is odd + odd + odd $=$ odd

Two shady characters, Rowan and Colin, approach you, claiming to have a large supply of these impossible squares. When you ask to see one, Rowan says: "No, it doesn't work like that. For each of our squares, I will reveal one row, and Colin one column. But you can choose which row and which column you want to know." You reply: "Do you think I was born yesterday? In each instance, Rowan can say any of his four possible answers $(001,010,100$ or 111 ) and Colin can choose whichever of his $(000,011,101$ or 110$)$ do not conflict with Rowan's. For example, if I ask for the second row and the third column, and Rowan says '001', then Colin just has to choose an answer with 1 as the middle entry, either 011 or $110 . "$

But Colin persists: "What if you prevent me from hearing not only Rowan's answer, but even the question put to Rowan? Take us far apart, and lock us in rooms that shield all forms of communication. We will still give consistent answers $100 \%$ of the time." You think to yourself: "In this case, their best strategy would be to each carry (or memorize) a predetermined list of answers to all possible questions. In a given trial, the respective answers that Rowan and Colin carry would have to correspond to squares that differ in at least one of the nine entries, because of the constraints on the rows and columns. If I conduct enough trials, choosing the questions at random, I will catch them with inconsistent answers soon enough."

So you agree to the trial as suggested; you ask questions in one room and an assistant in the other. To your consternation, Colin and Rowan give consistent answers every time. How is this possible? Are they communicating, despite all your efforts? No, they are using pairs of 'entangled' quantum particles - each pair of particles was jointly prepared in the same way, and then one kept by Rowan and one by Colin. With each trial, Rowan picks the next particle in his store, measures one of three different properties (depending on which row you ask for), and gives you one of his four possible answers based on the result of his measurement. Colin similarly processes his next particle, the one paired with Rowan's. By the 'magic' of quantum entanglement, their results are correlated precisely so as to simulate an impossible square ${ }^{10}$.

The moral (Bell's theorem): quantum correlations falsify the hypothesis that, in any laboratory, nature carries the answer to any question which may be put there, and answers without knowing which questions are being put elsewhere. H.W. and fast switching. It will be a landmark achievement in physics. But would such an experiment really close all the loopholes? The answer depends on one's attitude to causation.

The issue is whether the settings in one laboratory are uncorrelated with variables (hidden or otherwise) in the other. If they are correlated, then the experiment violates the assumptions of Bell's theorem, opening the free-choice loophole, so called because of how it can be closed: the only things correlated with free choices are their effects, so (by Einstein's principle) settings that are freely chosen late enough would be uncorrelated with the other variables, as desired.

Human choice and action are slow, so Bell experiments thus far have used random-number generators rather than free choice to change the detector settings. There is no reason for such random numbers to be correlated with anything on the other side. But if one is inclined to reject the principle of common cause (as localists are) then one must admit that correlations can occur without any reason. Thus, to be rigorous, experimenters must choose the settings freely.

Using human free-choice while closing the separation loophole would require separating the experimenters by much more than one Earth diameter (only 40 lightmilliseconds). Putting one experimenter on the Moon (1.3 lightseconds away) would also allow time for them to consciously register the results - a requirement to rule out a fourth and final loophole, the 'collapse loophole". This arises from the possibility that the set of potential results recorded by a detector does not 'collapse' to an actual individual result until observed by the experimenter, so that before the experimenter gets involved the result could be influenced, long after the photon arrives, by some bizarre (but not faster-than-light) causal influence from the distant laboratory.

Such an Earth-Moon experiment is a worthy challenge for the next 50 years.

Howard Wiseman is professor of physics at the Centre for Quantum Dynamics, Griffith University, Brisbane, Queensland, Australia. e-mail:h.wiseman@griffith.edu.au

1. Bell, J. S. Physics 1, 195-200 (1964).

2. Aspect, A., Dalibard, J. \& Roger, G. Phys. Rev. Lett. 49, 1804-1807 (1982)

3. Barrett, J., Hardy, L. \& Kent, A. Phys. Rev. Lett. 95, 010503 (2005)

4. Maudlin, T. Quantum Non-Locality \& Relativity (Blackwell, 2011)

5. Wiseman, H. M. Preprint at http://arxiv.org/ abs/1402.0351 (2014)

6. Bell, J. S. Epistemol. Lett. 9, 11-24 (1976).

7. Giustina, M. et al. Nature 497, 227-230 (2013)

8. Christensen, B. G. et al. Phys. Rev. Lett. 111, 130406 (2013)

9. Kent, A. Phys. Rev. A 72, 012107 (2005).

10.Aravind, P. K. Found. Phys. Lett. 15, 397-405 (2002). 